\title{
Cone-beam Computed Tomographic Analysis of C-shaped Canals and Radicular Grooves in Mandibular Premolars: Prevalence and Related Factors
}

\author{
Swati Srivastava ${ }^{1}$, Rahul N Gaikwad ${ }^{2}$, Noorah Alsalhi ${ }^{3}$, Noura A Alrogaibah ${ }^{4}$
}

\begin{abstract}
Aim: This study aimed to evaluate the prevalence of C-shaped canals and radicular grooves (RGs) in mandibular premolars using cone-beam computed tomography (CBCT) and correlating it with other factors like age, sex, unilateral, and bilateral prevalence.

Materials and methods: CBCT images of 242 patients with an age group of 13-70 years were selected from pre-existing database. The prevalence of C-shaped canal and RG was recorded along with variables such as age, sex, and bilateral prevalence.

Statistical analysis: Prevalence was compared using the Chi-square test $(p<0.05)$.

Results: The prevalence of C-shaped configurations in the mandibular first premolars (17.4\%) was significantly higher $(p<0.05)$ as compared with the mandibular second premolars (7.4\%). CBCT analysis revealed that in cervical third, $88 \%$ of canals were found to be C4 type. A high prevalence of C2 type canal was found in middle (68.6\%) and apical third (31.3\%). The prevalence of RG in the mandibular first premolars (13\%) was significantly higher $(p<0.05)$ as compared with the mandibular second premolars $(4.3 \%)$. There was no statistically significant difference between sex and prevalence of C-shaped canals and RG but it was significantly higher in the age group of $<50$ years $(p<0.05)$.

Conclusion: This study showed a high prevalence of C-shaped canals and RG in mandibular first premolars as compared with mandibular second premolars. The prevalence of the C-shaped canal and RG was significantly higher in the age group of $<50$ years. This knowledge may aid the clinicians for good endodontic prognosis.

Clinical significance: This study result provides valuable insight into the prevalence of C-shaped canal and RG among the premolars which can help clinicians to prevent perforations or root fractures.

Keywords: Cone-beam computed tomography, C-shaped canal, Mandibular premolars, Radicular groove.

The Journal of Contemporary Dental Practice (2019): 10.5005/jp-journals-10024-2692
\end{abstract}

\section{INTRODUCTION}

Morphological variations of root canal systems can be a challenge for root canal cleaning, shaping, and obturation, which may imperil the endodontic treatment outcome. Endodontic management of C-shaped anatomy is an interesting and challenging procedure and its success depends on individual skills and techniques applied.

In the past two decades, the literature has reported the C-shaped root canal anatomy in both maxillary and mandibular molars and premolars. According to slowey, mandibular premolars possess the greatest anatomical challenges with respect to accomplishing successful treatment outcome during root canal therapy. ${ }^{1} \mathrm{C}$-shaped canal system is associated with fins and isthmus connecting individual canals, which would change the cross-sectional canal morphology from orifice to apex. It may leave dead space and increase the difficulty during root canal treatment. The C-shaped anatomy gives rise to radicular groove (RG) in mandibular premolars, which result in the $\mathrm{C}$-shaped root cross section. ${ }^{2}$ Clinically, they may act as a reservoir for dental plaque and calculus, increasing the difficulty for the management of periodontal disease.

Recently, CBCT has been used as a reliable aid in the identification of root canal system configurations with superior sensitivity relative to two-dimensional radiographic modalities. Differences in gender and bilateral prevalence of anatomic variations can be compared easily on in vivo CBCT images because of their certain identification of tooth position and study subjects. ${ }^{3}$

The literature lacks detailed data of anatomy of mandibular premolars based on geographic and ethnic patterns especially
1,3,4 Department of Conservative Dental Sciences, College of Dentistry, Qassim University, Buraydah, Kingdom of Saudi Arabia

${ }^{2}$ Department of Community Dentistry and Oral Epidemiology, College of Dentistry, Qassim University, Buraydah, Kingdom of Saudi Arabia

Corresponding Author: Swati Srivastava, Department of Conservative Dental Sciences, College of Dentistry, Qassim University, Buraydah, Kingdom of Saudi Arabia, Phone: +966 531645063, e-mail: swatisrivastava412@gmail.com

How to cite this article: Srivastava S, Gaikwad RN, Alsalhi N, et al. Cone-beam Computed Tomographic Analysis of C-shaped Canals and Radicular Grooves in Mandibular Premolars: Prevalence and Related Factors. J Contemp Dent Pract 2019;20(11):1350-1354.

Source of support: Nil

Conflict of interest: None

in African, Australian, Asian, and South American populations. Presently, there is very limited information regarding the true prevalence of the C-shaped canals and RG in mandibular premolars. Till now, no such work has been carried out in Saudi population using cone-beam computed tomography (CBCT). Hence, in this research, the primary outcome was to evaluate the prevalence of C-shaped canal configurations and RG in mandibular first and second premolars using CBCT in Saudi subpopulation. The secondary outcomes were correlating it with other factors like age, sex, unilateral, and bilateral prevalence. 


\section{Materials and Methods}

\section{Image Selection}

This study was approved by the Dental Research Ethics Committee (Code: EA/6017/2018) by the Dental Research Center, College of Dentistry, Department of Conservative Dental Science, Qassim University, Kingdom of Saudi Arabia. Sample calculation was performed using $95 \%$ confidence interval so as to have a precision of $5 \%$. CBCT images of 242 patients with an age group of $13-70$ years were retrieved from an encrypted CBCT database between February 2016 and February 2019. Inclusion criteria for CBCT images were fully erupted permanent first and second mandibular premolars with mature apex and high-resolution images. Exclusion criteria were open apices, root resorption, calcifications, root canal treatments, posts, crowns, developmental disorders, pathologies, and history of orthodontic treatment.

Out of 242 cases, 156 cases ( 74 females and 82 males, with a mean age of 38.5 years) fulfilled the inclusion criteria. In these 156 cases, 276 first mandibular premolars and 258 second mandibular premolar images were analyzed.

\section{Imaging Method}

The CBCT unit used in this study was GALILEOS Comfort (DentsplySirona Dental Systems, Montagematerial, Galileos, SK, Bensheim, Germany). It had a tube voltage of $85 \mathrm{kVp}$; a tube current of 5-7 mA; a field of view of $15 \times 15 \times 15 \mathrm{~cm}^{3}$; an isotropic voxel size of $0.3-0.15 \mathrm{~mm}$; and an exposure time of 14 seconds to $2-6$ seconds. Images were examined by using the scanner's proprietary software (Sidexis XG 3D Viewer; Germany) in an Intel Core i5-4460 at $3.20 \mathrm{GHz}$ (Intel Corp, Santa Clara, CA) PC workstation running Windows XP professional SP-2 (Microsoft Corp, Redmond, WA). The level of the images was adjusted using the image processing tool in the software to ensure optimal visualization.

\section{Imaging Analysis}

Two observers, an oral and maxillofacial radiologist and an endodontist, analyzed the images simultaneously to reach an agreement for the interpretation of the CBCT images. The assessment of mandibular first and second permanent premolars was done in three planes (axial, sagittal, and cross-sectional) at $1.0 \mathrm{~mm}$ intervals, by continuously moving the toolbar from the floor of the pulp chamber to the apex. Patients were divided based on sex and into three age groups: $13-30,31-50$, and $51-70$ years. In an axial view, presence of a RG was analyzed according to Fan et al. ${ }^{4}$ as deep groove or shallow groove. The C-shaped root canal anatomy was analyzed according to Fan et al. in the cervical, middle, and apical third as follows:

- Category I (C1): the canal shape was an uninterrupted " $\mathrm{C}$ " with no other separations or divisions.

- Category II (C2): the canal shape resembled a semicolon resulting from a discontinuation in the " $\mathrm{C}$ " outline.

- Category III (C3): two separate round, oval, or flat canals.

- Category IV (C4): only one round, oval, or flat canal.

Images were also analyzed for age, unilateral, and bilateral prevalence of both RG and C-shaped canals.

\section{Statistical Analysis}

Data analysis was performed with the help of the Statistical Package for Social Sciences Version 22 (SPSS Inc., Chicago, IL, USA). Differences in the prevalence of the C-shaped canal and RG based on sex, age, and bilateral prevalence were compared using the Chi-square test with a level of significance as $p<0.05$.

\section{Results}

An analysis of 156 cases was carried out initially. A total of 34 cases were finalized having C-shaped and 24 cases for RG.

\section{C-shaped Canals}

In 276 first mandibular premolars, 48 teeth had C-shaped configuration. The prevalence of mandibular first premolar with C-shaped canal was $17.4 \%$. In 258 second mandibular premolars, 19 teeth had C-shaped configuration. The prevalence of mandibular second premolar with C-shaped canal was 7.4\%. Total prevalence of C-shaped canals in mandibular premolars was $12.5 \%$. The prevalence of $\mathrm{C}$-shaped configurations in the mandibular first premolars was significantly higher $(p<0.05)$ as compared with the mandibular second premolars (Table 1). During analysis of axial slices in the cervical third, $88 \%$ of canals were found to be $\mathrm{C} 4$ type. A high prevalence of C2 type canal was found in middle and apical third (68.6\% and $31.3 \%$, respectively). The prevalence of C1 type canal was $23.9 \%$ in middle third and $18 \%$ in apical third. The prevalence of C3 type canal was $11.9 \%$ in cervical third, $7.5 \%$ in middle third, and $28.3 \%$ in apical third (Table 2; Figs 1 and 2).

\section{RG}

In 276 first mandibular premolars, 36 teeth had RG. The prevalence of mandibular first premolar with RG was 13\%. In 258 second mandibular premolars, 11 teeth had RG. The prevalence of mandibular second premolar with RG was $4.3 \%$. Total prevalence of RG in mandibular premolars was $8.8 \%$. The prevalence of RG in the mandibular first premolars was significantly higher $(p<0.05)$ as compared with the mandibular second premolars (Table 1). Among 47 premolars having RG, 18 (38.3\%) had deep mesiolingual RGs and $29(61.7 \%)$ had a shallow depression on proximal sides.

\section{Age and Sex}

There was no statistically significant difference between sex and prevalence of C-shaped canals and RG but it was significantly higher in the age group of $<50$ years $(p<0.05)$ (Table 3 ).

\section{Bilateral Prevalence}

Out of 156 cases, $23(14.7 \%)$ cases had bilateral prevalence of C-shaped canals, 11 (7\%) cases had unilateral occurrence, and the remaining 122 (78.2\%) cases had no C-shaped canals in mandibular premolars. Regarding RG, 18 (11.5\%) cases had bilateral prevalence, $6(3.8 \%)$ had unilateral occurrence, and the remaining 132 (84.6\%) cases had no RG in mandibular premolars (Table 4).

Table 1: Number $(n)$ and percentage (\%) of radicular groove and C-shaped canal in mandibular first and second premolars. Different superscript uppercases in the same column indicate statistically significant difference $(p<0.05)$

\begin{tabular}{llll}
\hline Tooth & Sample size & $\begin{array}{l}\text { C-shaped canal } \\
n(\%)\end{array}$ & $\begin{array}{l}\text { Radicular } \\
\text { grooven }(\%)\end{array}$ \\
\hline $\begin{array}{l}\text { Mandibular first } \\
\text { premolar }\end{array}$ & 276 & $48(17.4 \%)^{\mathrm{A}}$ & $36(13 \%)^{\mathrm{A}}$ \\
$\begin{array}{l}\text { Mandibular } \\
\text { second premolar }\end{array}$ & 258 & $19(7.4 \%)^{\mathrm{B}}$ & $11(4.3 \%)^{\mathrm{B}}$ \\
\begin{tabular}{l} 
Total \\
\hline
\end{tabular} & 534 & $67(12.5 \%)$ & $47(8.8 \%)$ \\
\hline
\end{tabular}


Table 2: Number ( $n$ ) and percentage (\%) of C-shaped canal configurations of mandibular premolars in different axial slices

\begin{tabular}{llllll}
\hline & C1 type & C2 type & C3 type & C4 type & Total \\
\hline Cervical third & - & - & $8(11.9 \%)$ & $59(88 \%)$ & $67(100)$ \\
Middle third & $16(23.9 \%)$ & $46(68.6 \%)$ & $5(7.5 \%)$ & - & $67(100)$ \\
Apical third & $12(18 \%)$ & $21(31.3 \%)$ & $19(28.3 \%)$ & $722.4 \%)$ & $67(100)$ \\
Total $n(\%)$ & $28(13.9 \%)$ & $67(33.3 \%)$ & $32(16 \%)$ & $74(36.8 \%)$ & $201(100)$ \\
\hline
\end{tabular}
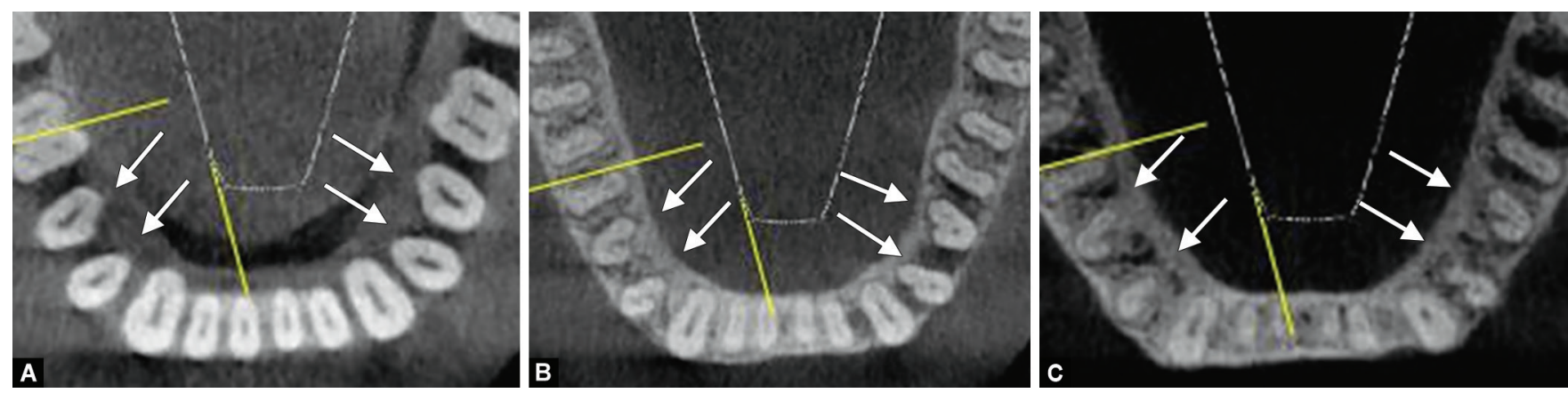

Figs 1 A to C: Bilateral prevalence of C-shaped canal and RG in same case in all premolars: (A) Shallow RG and C4 type C-shaped canal in cervical third; (B) Deep RG, C2 type C-shaped canal in 44 and 45 and C3 type C-shaped canal in 34, 35 in middle third; (C) Deep RG, C3 type C-shaped canal in apical third
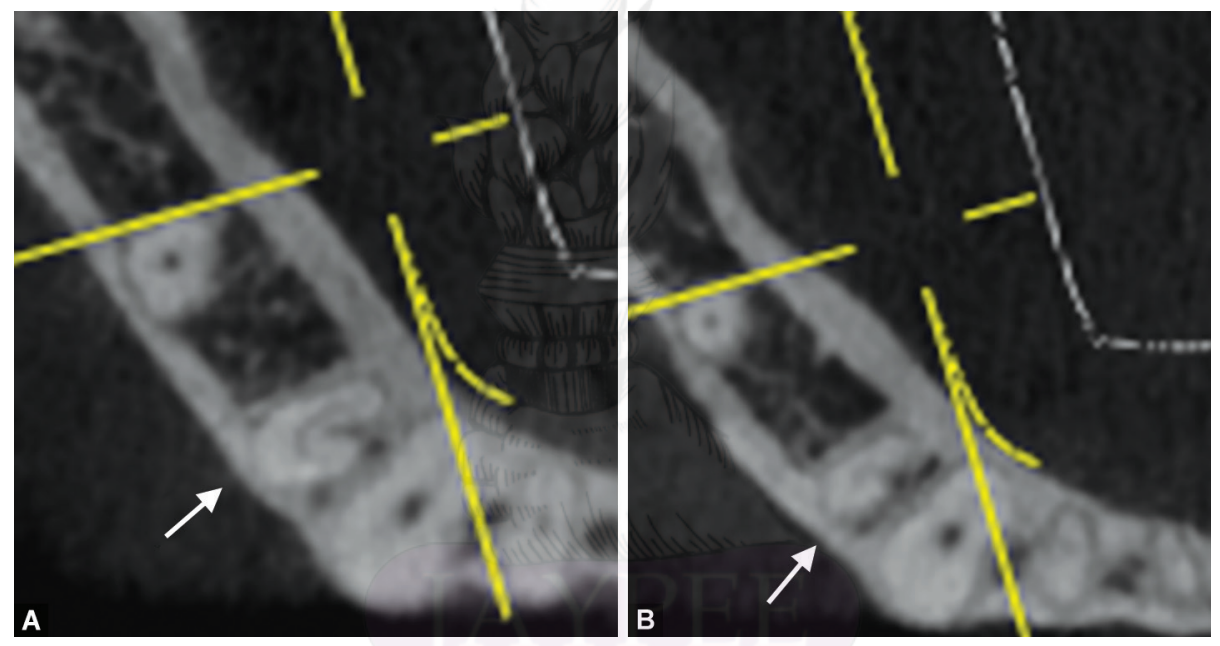

Figs 2A and B: C1 type C-shaped canal: (A) In middle third; (B) In apical third

Table 3: Number ( $n$ ) and percentage (\%) of C-shaped canal and RG in different age groups. Different superscript uppercases in the same column indicate statistically significant difference $(p<0.05)$

\begin{tabular}{lll}
\hline Age (years) & C-shaped canal $n(\%)$ & $R G n(\%)$ \\
\hline$<50$ & $28(82.3 \%)^{\mathrm{A}}$ & $17(70.8 \%)^{\mathrm{A}}$ \\
$\geq 50$ & $6(17.6 \%)^{\mathrm{B}}$ & $7(29.2 \%)^{\mathrm{B}}$ \\
Total & 34 & 24 \\
\hline
\end{tabular}

Table 4: Number ( $n$ ) and percentage (\%) of bilateral and unilateral prevalence of C-shaped canal and RG in mandibular premolars

\begin{tabular}{llll}
\hline & $\begin{array}{l}\text { Bilateral } \\
\text { prevalence }\end{array}(\%)$ & $\begin{array}{l}\text { Unilateral } \\
\text { prevalence n (\%) }\end{array}$ & Neither n (\%) \\
\hline C-shaped canal & $23(14.7 \%)$ & $11(7 \%)$ & $122(78.2 \%)$ \\
RG & $18(11.5 \%)$ & $6(3.8 \%)$ & $132(84.6 \%)$ \\
\hline
\end{tabular}

\section{Discussion}

C-shaped root canal system of mandibular premolars is a complex anatomy and endodontically challenging. It is characterized by the presence of isthmus, fins, and root canals merging. ${ }^{5}$ Identification of C-shaped anatomy is more challenging as it presents itself as a single oval canal in cervical third predominantly and the C-shaped anatomy begins at the middle third. ${ }^{6}$

CBCT imaging has outperformed regular radiography in terms of its ability for detecting and interpreting aberrant root canal morphology. ${ }^{7}$ It has the advantage of a significantly lower radiation dose with a higher resolution. This study required CBCT scans of patients without exposing them to radiations. Hence, we accessed an already existing database in oral and maxillofacial radiology department.

C-shaped canal configuration is believed to be due to failure of Hertwig's epithelial root sheath to fuse during the tooth 
development or coalescence by the continuous cementum deposition. A gene located on chromosome 5 in mice has also been reported to be the most likely candidate gene to cause C-shaped roots. ${ }^{8}$ Patients with X-linked human inherited diseases also showed increased additional root formation in mandibular premolars, suggesting that the genetic change on $\mathrm{X}$ chromosome may be a factor contributing to the $\mathrm{C}$-shaped root formation. ${ }^{9}$ These complex anatomic inconsistencies may expand the difficulties amid endodontic treatment.

In this study, we used Fan et al. classification ${ }^{6}$ based on microcomputed tomography to describe the C-shaped anatomy. He described C-shaped root canal as a cross- sectional root canal system presenting a continuous " $c$ " with no separation or division (C1); a canal shape resembling a semicolon resulting from a discontinuation in the " $\mathrm{C}$ " outline (C2); two separate round, oval, or flat canals (C3); and only one round, oval, or flat canal (C4).

In general, the prevalence of C-shaped root canal morphology in mandibular first premolars is reported to range from $1.1 \%$ to $18 \% .^{10-12}$ Alkaabi et al. found $28 \%$ prevalence of C-shaped canal in an Emirati population. ${ }^{13}$ We found a significantly higher prevalence (17.4\%) in mandibular first premolars than as compared to mandibular second premolars (7.4\%). C-shaped root canal morphology in mandibular second premolars is reported to be rare. ${ }^{11}$ Their prevalence seems to have a smaller variation among different populations, perhaps due to the very low prevalence. ${ }^{11,12}$

The results of the present study showed a high prevalence $(12.5 \%)$ of mandibular premolar C-shaped anatomy in Saudi subpopulation. The variations in results might be attributed to different ethnic groups, study design, sample size, and method of inspection.

During the serial axial $C B C T$ image examination, we found that $\mathrm{C}$-shaped canals could change to $\mathrm{C} 1, \mathrm{C} 2$, and $\mathrm{C} 3$ from the middle and apical third. Eighty-eight percent of canals were C4 type in cervical third and a high prevalence of C2 type was found in middle and apical third (68.6\% and $31.3 \%$, respectively). Our results are in line with previous studies done by Fan et al. and Ordinola-Zapata et al. ${ }^{14}$ who via microcomputed tomography stated that the coronal third presented mainly cross-sections with one single round or oval root canal. The more complex $\mathrm{C} 1$ and $\mathrm{C} 2$ configurations were present mainly in the middle third, whereas the apical section had a majority of C2 or two independent oval or round root canals.

We used the classification of RG given by Fan et al. ${ }^{4}$ dividing them into deep and shallow grooves. A "deep groove" was defined as the depth of the groove exceeding one-third of the bucco-lingual or mesio-distal dimension of the tooth. Whereas the other grooves were classified as "shallow grooves". We found prevalence of $8.8 \%$ for RG. Our results are lower as compared to other studies which might be attributed to the fact that prevalence of RGs shows ethnic predilection. ${ }^{5,12,15}$ In the present study, most of the RG were found on mesiolingual surface of the root. Our findings are in agreement with Lu et al. ${ }^{12}$ who found RG on the proximal lingual area of the middle root which did not always extend to the root apex.

For both C-shaped canal and RG, we did not find any significant difference between sex, but it was significantly higher in the age group of $<50$ years. In younger patients during tooth development, the irregular fusion of the Hertwig's epithelial sheath was attributed to trauma, such as radiation or chemical interference, but following the documentation of racial predilection, it is more likely to be of genetic origin. ${ }^{16,17}$ The root canals in such teeth merge in a very wide, slotlike, single, continuous root canal morphology, which can assume the shape of a letter $C .^{18}$ We found bilateral prevalence of $14.7 \%$ for C-shaped canals and $11.5 \%$ for RG which varies according to different racial populations investigated.

In this study, there were some limitations. First, only high resolution CBCT images were considered for analysis. Second, the teeth with exclusion criteria like obturated canals, open apices, calcified canals, and presence of artifact images which could interfere with the correct analysis were not analyzed. These excluded samples were uncommon and a large sample size would have minimized its effect in the final prevalence results. Third, the results may vary based on ethnicity, study design, and sample size.

\section{Conclusion}

The clinician must be careful to avoid strip perforation due to vigorous instrumentation in thin roots of C-shaped mandibular premolars. Use of CBCT and dental operating microscope may aid in avoiding it and improving endodontic outcome. The presence of RG dictates a C-shaped root cross section. RG can complicate restorations with posts and root canal instrumentation and obturation. To prevent perforation or root fracture, clinicians should understand the correlation between the isthmus wall thickness and RG depth especially in mandibular first premolars.

\section{Author Contribution}

All authors have contributed equally.

\section{ACKnowledgment}

The authors would like to thank the Oral and Maxillofacial Radiology Department, College of Dentistry, Qassim University, Kingdom of Saudi Arabia.

\section{REFERENCES}

1. Slowey RR. Root canal anatomy. Road map to successful endodontics. Dent Clin North Am 1979;23(4):555-573.

2. Gu YC, Zhang YP, Liao ZG, et al. A micro-computed tomographic analysis of wall thickness of C-shaped canals in mandibular first premolars. J Endod 2013;39(8):973-976. DOI: 10.1016/ j.joen.2013.04.039.

3. Huang RY, Cheng WC, Chen CJ, et al. Three-dimensional analysis of the root morphology of mandibular first molars with distolingual roots. Int Endod J 2010;43(6):478-484. DOI: 10.1111/j.1365-2591.2010. 01702.x.

4. Fan B, Ye W, Xie E, et al. Three-dimensional morphological analysis of C-shaped canals in mandibular first premolars in a Chinese population. Int Endod J 2012;45(11):1035-1041. DOI: 10.1111/j.13652591.2012.02070.x.

5. Jafarzadeh $\mathrm{H}, \mathrm{Wu}$ YN. The C-shaped root canal configuration: a review. J Endod 2007;33(5):517-523. DOI: 10.1016/j.joen.2007. 01.005 .

6. Fan B, Yang J, Gutmann JL. Root canal systems in mandibular first premolars with C-shaped root configurations. Part I: Microcomputed tomography mapping of the radicular groove and associated root canal cross-sections. J Endod 2008;34(11):1337-1341. DOI: 10.1016/ j.joen.2008.08.006.

7. Cotton TP, Geisler TM, Holden DT, et al. Endodontic applications of cone-beam volumetric tomography. J Endod 2007;33(9):1121-1132. DOI: 10.1016/j.joen.2007.06.011.

8. Tashima I, Arita K, Asada Y. Genetic study of gutter-shaped root (GSR) in AKXL RI mouse strains using QTL analysis. J Oral Sci 2010;52(2): 213-220. DOI: 10.2334/josnusd.52.213. 
9. Kusiak A, Sadlak-Nowicka J, Limon J, et al. Root morphology of mandibular premolars in 40 patients with Turner syndrome. Int Endod J 2005;38(11):822-826. DOI: 10.1111/j.1365-2591.2005.01023.x.

10. Awawdeh LA, Al-Qudah AA. Root form and canal morphology of mandibular premolars in a Jordanian population. Int Endod J 2008;41(3):240-248. DOI: 10.1111/j.1365-2591.2007.01348.x.

11. Yu X, Guo B, Li KZ, et al. Cone-beam computed tomography study of root and canal morphology of mandibular premolars in a western Chinese population. BMC Med Imaging 2012;12:18. DOI: 10.1186/14712342-12-18.

12. Lu TY, Yang SF, Pai SF. Complicated root canal morphology of mandibular first premolar in a Chinese population using the cross section method. J Endod 2006;32(10):932-936. DOI: 10.1016/ j.joen.2006.04.008

13. Alkaabi W, AlShwaimi E, Farooql, et al. A micro-computed tomography study of the root canal morphology of mandibular first premolars in an emirati population. Med Princ Pract 2017;26(2):118-124. DOI: $10.1159 / 000453039$.
14. Ordinola-Zapata R, Monteiro Bramante $C$, Gagliardi Minotti $P$, et al. Micro-CT evaluation of C-shaped mandibular first premolars in a Brazilian subpopulation. Int Endod J 2015;48(8):807-813. DOI: 10.1111/ iej.12380.

15. Chen J, Li X, Su Y, et al. A micro-computed tomography study of the relationship between radicular grooves and root canal morphology in mandibular first premolars. Clin Oral Investig 2015;19(2):329-334. DOI: 10.1007/s00784-014-1242-1.

16. Manning SA. Root canal anatomy of mandibular second molars. Part II. C-shaped canals. Int Endod J 1990;23(1):40-45. DOI: 10.1111/j.13652591.1990.tb00801.x.

17. Fischischweiger W, Clausnitzer E. Root formation in molar teeth of the CD-1 mouse. J Endod 1988;14(4):163-168. DOI: 10.1016/S00992399(88)80256-5.

18. Carlsen O, Alexandersen V, Heitmann T, et al. Root canals in onerooted maxillary second molars. Scand J Dent Res 1992;100(5): 249-256. DOI: 10.1111/j.1600-0722.1992.tb01067.x. 